\title{
Medicean Moons Sailing Through Plasma Seas: Challenges in Establishing Magnetic Properties
}

\author{
Margaret G. Kivelson ${ }^{1,2}$, Xianzhe Jia ${ }^{2}$ and Krishan K. Khurana ${ }^{1}$ \\ ${ }^{1}$ Department of Earth and Space Sciences, University of California, \\ Los Angeles, CA 90095-1567,USA \\ email: mkivelson@igpp.ucla.edu \\ ${ }^{2}$ Department of Atmospheric, Oceanic and Space Sciences, University of Michigan, Ann Arbor, \\ MI 48109-2143
}

\begin{abstract}
Jupiter's moons, embedded in the magnetized, flowing plasma of Jupiter's magnetosphere, the plasma seas of the title, are fluids whose highly non-linear interactions imply complex behavior. In a plasma, magnetic fields couple widely separated regions; consequently plasma interactions are exceptionally sensitive to boundary conditions (often ill-specified). Perturbation fields arising from plasma currents greatly limit our ability to establish more than the dominant internal magnetic field of a moon. With a focus on Ganymede and a nod to Io, this paper discusses the complexity of plasma-moon interactions, explains how computer simulations have helped characterize the system and presents improved fits to Ganymede's internal field.
\end{abstract}

Keywords. Galilean moons, magnetic field, magnetosphere.

\section{Introduction}

Galileo would have been intrigued had he known that today's space explorers can tell us about the surfaces, interiors, atmospheres, and charged particle environments of his Medicean moons. The high resolution images provided by spacecraft have transformed his tiny points of light into worlds to be described by geologists and geophysicists. Relevant to this paper is the evidence that some of the moons are sources of magnetic fields, and that all of them are embedded in flowing, magnetized plasmas. Plasmas are highly ionized gases, on average electrically neutral but capable of conducting electrical currents. In the vicinity of a moon, the ambient plasma is diverted and otherwise perturbed. Those perturbations drive currents that can flow through the moons and their ionized environments and can also flow along Jupiter's background field to its ionosphere, its ionized upper atmosphere. The interactions are highly non-linear and are affected by properties of both local and remote parts of the system because of the currents that link them. This chapter emphasizes that the complexity introduced by the presence of plasma makes it challenging to characterize the internal magnetic properties of a moon from spacecraft measurements. Using Ganymede as an example, this paper describes a method of removing magnetic signatures generated within the ambient plasma from spacecraft measurements of the magnetic field in the vicinity of a moon. The remaining signature can be attributed to currents flowing within the moon and therefore provides information about the moon's interior.

In seeking to understand the interior structure of a moon, remote measurements provide little insight. Taking Ganymede as an example, Pioneer and Voyager were able to measure its mass, establishing an average density $\left(1,940 \mathrm{~kg} / \mathrm{m}^{-3}\right.$ taken to be roughly 
$60 \%$ rock and $40 \%$ ice), but did not reveal whether the interior is relatively homogeneous or differentiated. Early models of the interiors of the icy moons typically consider only a rocky core and an ice-silicate shell (e.g., Schubert et al., 1981). Only after Galileo acquired data from relatively low altitude flybys and established higher order gravitational moments did it become clear that the deep interior of Ganymede is differentiated into a metallic core and a rocky mantle (Anderson et al., 1996). Although invaluable for establishing the distribution of mass within the interior of a moon, the gravitational moments are insensitive to the state of the matter forming the zones of differing density, and it is here that knowledge of magnetic properties becomes critical. Magnetic moments give insight into the distribution of conducting matter in the interior. For differentiated bodies like the moons of Jupiter, the dense cores are conductors, but only if they are partially fluid can dynamo action generate an internal field. Inductive fields can arise in any highly conducting layer exposed to time-varying external magnetic fields. The discussion below deals with both dynamo fields and induced fields and provides arguments supporting the view that induced fields (identified at Europa and Callisto as well as Ganymede) require warm (probably melted) layers within the icy outer shells. Thus the primary clues to the physical state of a moon's interior are provided by measurements of the magnetic moments and their temporal variation.

Because the magnetic moments provide insights that cannot be obtained from other measurements, it is important that their values be established accurately. The next section describes why refined estimates of the magnetic moments of a moon require knowledge of the contribution of plasma currents to the total field.

\section{Problems in characterizing the internal magnetic field of a moon}

A moon's internal magnetic moments are characterized by modeling the field measured in the vicinity of the moon as the sum of fields arising from internal and external currents. The problem is well posed if measurements are available over a current-free closed surface surrounding the moon. In this case, the internal and external multipole moments can be obtained by following a prescription that goes back to Gauss (Walker and Russell, 1995). However, measurements at the moons of Jupiter are sparse, and do not even approximately provide measurements over a closed surface. For example, at distances closer than $2 \mathrm{R}_{G}\left(\mathrm{R}_{G}\right.$ is the radius of Ganymede, $\left.2631 \mathrm{~km}\right)$ from the center of

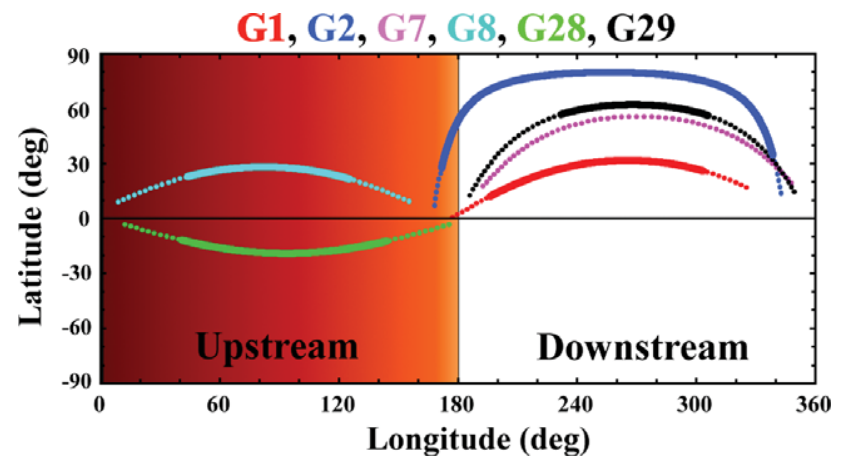

Figure 1. Latitude and longitude relative to a Ganymede-centered spherical coordinate system of the Galileo trajectories during the six close flybys of Ganymede. The portions of Galileo flyby trajectories closer than $2 \mathrm{R}_{G}$ to the center of Ganymede are represented by the solid traces. Latitude is measured relative to the spin equator and longitude is measured in a right hand sense relative to $0^{\circ}$ in the Jupiter-facing meridian. 
Ganymede, there are no measurements south of $20^{\circ}$ latitude and in one quadrant there are no measurements at all (see Figure 1). Furthermore, strong currents flow throughout the near neighborhoods of the moons, so the available measurements are made in regions that are not current-free. Finally, the modeled sources referred to as internal include sources that are below the spacecraft trajectory but may be above the surface of the moon (see Figure 2). Currents flow above the surfaces because the gases that fill the immense Jovian magnetosphere are, for the most part, electrically charged but, because in any significant volume the charges balance, the system remains electrically neutral. Spatial changes of the magnetic field generate currents (through $\nabla \times \mathbf{B}=\mu_{\mathbf{o}} \mathbf{j}$ ), while temporal changes produce inductive electric fields (through $\partial \mathbf{B} / \partial \mathbf{t}=-\nabla \times \mathbf{E}$ ) and associated currents. Here $\mathbf{B}(\mathbf{E})$ is the magnetic (electric) field, $\mathbf{j}$ is the current, and $\mu_{o}$ is the permeability of vacuum.

In considering the interaction of the gas of charged particles, a plasma, with the Medicean moons, it is useful to describe how currents arise and how particle flows and magnetic fields relate. Magnetospheric plasmas are tenuous; collisions are infrequent and particles interact principally through electromagnetic forces. Ions and electrons respond differently to the imposed forces and their differing motions produce net current. In response to the Lorentz force $(\mathbf{F}=q \mathbf{v} \times \mathbf{B}$ with $q$ the particle charge and $\mathbf{v}$ its velocity $)$ that acts perpendicular to the magnetic field, the plasma particles move across the background field on quasi-circular orbits (for a particle of mass $m$ and perpendicular velocity $v_{\perp}$, the radius is $m v_{\perp} / q B$ with the sense of rotation depending on the sign of the charge). The Lorentz force has no component along the field, so charged particles respond only to field gradients in their field-aligned motion. The consequence is that charged particles bounce back and forth along $\mathbf{B}$ following spiraling paths with small transverse displacements. It is convenient to think of magnetic field lines loaded with spiraling charged particles as identifiable structures that can be followed as they move through space, and to assert

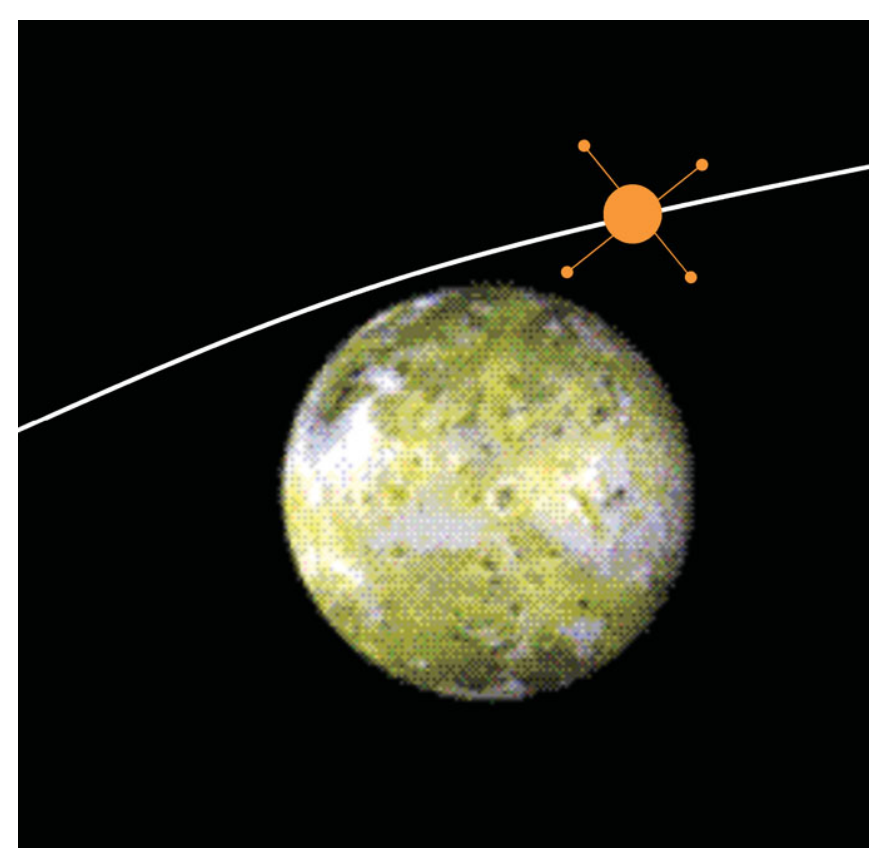

Figure 2. Schematic flyby of a moon. Currents flowing below the trajectory, whether inside the moon or above its surface, contribute as "internal" sources. 
that the field and plasma are "frozen" together. It then follows that particle flows can displace and distort field lines. We will make use of this intuitive language in discussing the interaction of the magnetized plasma of the Jovian magnetosphere and the Galilean moons.

\section{The plasma of Jupiter's magnetosphere}

The structure of the plasma environment of a moon depends on the properties of the magnetized plasma in which it is embedded, but specifics of the interaction are controlled by the physical properties of the moon itself (Kivelson, 2004; Jia et al., 2009b). The plasma relevant to describing interactions with the moons is the magnetospheric plasma trapped in Jupiter's strong magnetic field, dominated by a dipole moment tilted by about $10^{\circ}$ relative to the spin axis. Over each Jupiter rotation period ( $\sim 10$ hours $)$, the magnetic field imposed in the neighborhood of a moon is oriented dominantly southward but tilts radially inward and outward. The variation of the radial field occurs at the synodic period (the period of Jupiter's rotation as observed in the moon's rest frame), which is slightly longer than 10 hours because of the moon's orbital motion. The magnetospheric plasma in which the moon is embedded flows azimuthally, maintained in motion by field-aligned currents coupled to the planet. The angular speed is close to full corotation with Jupiter. Beyond $\sim 2 \mathrm{R}_{J}$ the speed of corotation is faster than Keplerian, so plasma overtakes all of the moons, flowing towards them from their trailing sides. [The Medicean moons move in nearly circular orbits in Jupiter's equatorial plane at distances ranging from $\sim 6 \mathrm{R}_{J}$ for Io to $\sim 25 \mathrm{R}_{J}$ for Callisto $\left(\mathrm{R}_{J}\right.$ is Jupiter's radius, $\left.\sim 71,500 \mathrm{~km}\right)$.] Because plasma is concentrated close to the tilted magnetic equator, planetary rotation introduces additional slow north-south motions of the plasma relative to the rest frame of a moon.

If one of the moons of interest were inert and non-conducting, it would intercept and absorb the plasma flowing onto its cross section and a nearly empty wake would form in the region downstream in the plasma flow. This type of interaction approximates the situation at Earth's moon interacting with solar wind plasma. But the interaction of the plasma with Jupiter's moons and their surrounding ionospheres is quite different. Io, Europa, and Callisto (and their relatively dense ionized surroundings) are electrically conducting. Induced currents slow and divert much of the plasma flowing towards these moons so that only a small part of the upstream plasma flowing towards the cross-section of a moon actually reaches its surface. Because the field is frozen-in to the plasma, slowed flow on only a part of a flux tube (the part close to the moon) introduces a kink in the field. The associated field bending requires current to flow along the field and, in steady state, a bundle of perturbed flux tubes, referred to as an Alfvén wing, develops and moves through the plasma as if attached to the moon (Figure 3). Such structures and the currents that maintain them are of intrinsic interest (see, for example, Kivelson, 2004) but this paper focuses on plasma perturbations only to the extent that they mask the signatures of magnetic sources within the moons. This means that the distortion of the field produced by plasma currents, including those that form the Alfvén wings and others that close through the ionosphere or the solid portions of a moon, must be established in order to separate out the field perturbations generated by currents that flow completely within the moon.

\section{Magnetic fields of the Medicean moons}

Most of the Medicean moons have internally generated fields small compared with the field imposed by Jupiter at their orbital distances. Unique among the moons, Ganymede 
has a substantial internal magnetic field and a plasma interaction region dominated by internal sources of magnetism (Kivelson et al., 1996, 1997). The existence and magnitude of the field were both generally unexpected. The scaling law of Busse (1976) had been used by Neubauer (1978) to predict plausible magnitudes of possible internally generated fields of all the Galilean moons, but before Ganymede's field was discovered it was generally thought that, over geological time, the moons would have cooled sufficiently for their interiors to have solidified and their dynamos to have shut down. Io, in a strong tidal resonance, was viewed as a possible exception, a case for which a planetary scale molten interior consistent with widespread volcanic activity and possible dynamo action could not be ruled out (Kivelson et al., 1997). Yet Galileo ultimately found no evidence for a significant dynamo-generated magnetic field at Io (Jia et al., 2009b) but discovered that Ganymede's internal field (of order $750 \mathrm{nT}$ at the equatorial surface) is almost four times larger than had been suggested by scaling laws (Kivelson et al., 1996, 1997). The large field appears to require dynamo action and a molten metallic layer in the deep interior. This discovery has led to suggestions that orbital migration took Ganymede through a tidal resonance at some time in the past billion years, with interactions strong enough to melt substantial portions of its metallic core (Showman and Malhotra, 1997).

Although Ganymede is the only moon with a large permanent magnetic field, the story differs when attention turns to temporally varying fields. If there is conducting material within a moon, the time-varying component of Jupiter's field (mostly radial in orientation) can induce an EMF that in turn drives a time-varying current that generates an (induced) magnetic field. Only if the conducting layer is global in scale and lies close to the surface will the induced field be comparable in magnitude to the time-varying field component that causes it. Galileo measurements detected magnetic perturbations consistent with inductive fields at Europa and Callisto (Khurana et al., 1998; Zimmer et al, 2000). The amplitudes of the perturbation fields and their fall-off with radial distance provided evidence of the global scale of the conducting layers in which they were generated. A uniform time-varying field generates a dipolar response from a spherically symmetric shell. The amplitude of the field induced in a conducting body depends on its conductivity. The limiting case, for high conductivity, is that of a dipole field whose polar field is equal and opposite to the driving field at the surface of the conducting shell. Away
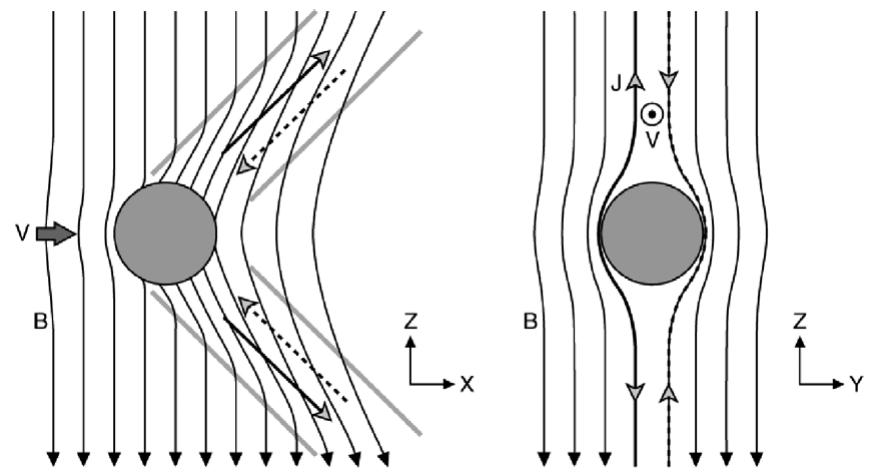

Figure 3. After Kivelson et al. (2001), Schematic of the Alfvén wing interaction. Lines with black arrowheads represent the magnetic field. Lines with gray arrowheads represent currents: those shown with dashed lines flow behind the plane of the image and those with solid lines flow in front of that plane. Light gray lines delimit the region in which perturbations linked to the Alfvén wing currents are significant. Left: A cross section through the center of the moon. Plasma flows to the right, transverse to the field. Right: A cross section orthogonal to the flow through the center of the moon. 
from the conducting shell (of radius $\mathrm{r}_{\text {shell }}$ ), the field strength falls as $\left(r_{\text {shell }} / r\right)^{3}$ where $r$ is the distance from the center of the shell. The large amplitude of the perturbations observed at both Europa and Callisto required the conducting layers for both moons to lie close to their surfaces, and consequently to be buried within the outer ice layers. Solid ice is not a good enough conductor to account for the observations, but a liquid water shell with electrolytes to carry current is consistent with the magnetometer measurements in both cases. For Ganymede, the case for a sub-surface ocean is less clear as the large dynamo-driven field and the surrounding magnetosphere complicate the signature. However, results of the most recent analysis again lead to the conclusion that Ganymede also contains a conducting sub-surface ocean (Jia, 2009).

\section{Interaction with the Jovian plasma}

Each moon interacts with the Jovian plasma in a slightly different way. The interactions greatly perturb the field in the neighborhood of the moon and on flux tubes linked magnetically to it. The regions modified by the interaction may be highly extended and highly structured. Significant perturbations arise from ionospheric currents and the plasma currents that link to them. Some moons (notably Io and to a lesser degree Europa) release neutrals into their environments, much like comets. The ionization of neutral matter generates what are referred to as pickup currents, which also perturb the magnetic field.

The interaction region is particularly complex in the vicinity of Ganymede whose strong internal field carves out a large cavity within the Jovian magnetospheric plasma, a minimagnetosphere whose boundary lies at distances greater than $1 \mathrm{R}_{G}$ above its surface. The cavity develops because magnetized plasmas do not intermingle except on extremely small length scales (of order the ion gyroradius) so Jupiter's plasma is diverted well above Ganymede's surface. Ganymede's magnetosphere, embedded in the sub-magnetosonic plasma flow of Jupiter's magnetosphere, differs in shape from magnetospheres of planets (e.g., Earth, Mercury, Jupiter and Saturn) that form in the super-magnetosonic flow of the solar wind. Planetary magnetospheres are bullet-shaped cavities aligned with the solar wind plasma flow. Ganymede's magnetosphere is quasi-cylindrical, about an axis aligned almost perpendicular to the plasma flow velocity and nearly parallel to the externally imposed field as illustrated in Figure $4(a$ and $b)$.

A magnetosphere is often described in terms of distinct current systems that account for its structure. Especially in the vicinity of boundaries such as the magnetopause across which the field may reverse direction over distances of a few ion gyroradii, magnetospheric currents can be intense. Data acquired in current-carrying regions compromise the process of obtaining fits to a moon's internal field, as noted in Section 2. For this reason, we next discuss improved numerical simulations of the interacting system that provide good estimates of the contributions of the external current system, contributions that can be removed in order to improve the estimate of the internal sources of magnetic field.

\section{Numerical simulations of the interaction region}

The interactions of the Jovian plasma and the moons of Jupiter have been modeled using a variety of numerical approaches. Each approach focuses on modeling some aspect of the physical system particularly well. All start by assuming a model of the internal field and the field of Jupiter's magnetosphere imposed at the location of the moon. Some prescribe the magnetic field and calculate the interaction of the plasma and the moon's ionosphere using a realistic model of its conductivity (Saur et al., 1998). The magnetic 
perturbations are calculated from the currents. This approach provides insight into the role of the ionosphere at the cost of self-consistency. Some follow ions and electrons separately, including small (ion gyroradii) scale contributions at the cost of higher spatial resolution (Paty and Winglee, 2006). Some use single fluid magnetohydrodynamics (MHD), ignoring multi-fluid and kinetics and simplifying the treatment of ionospheric conductivity (Schilling et al., 2007, 2008; Jia et al., 2008, 2009a). All have advantages and disadvantages and all can reproduce important features of the magnetic structure of the interactions.

Here we suggest that for a simulation to be useful for the purpose of establishing the internal field of a moon it must include:

- data-based upstream conditions;

- simulation boundaries that do not generate signals;

- physics-based boundary conditions at the moon's surface;

- appropriate handling of plasma resistivity to allow reconnection at a rate consistent with observations;

- output that faithfully reproduces all available measured properties of the system. Challenges arise in implementing the requirements noted. Unperturbed plasma and field measurements are available only somewhat before and after the actual encounter, so upstream plasma/field conditions must be extrapolated to the location and time of the encounter. Downstream boundaries do generate signals unless the run is truncated, so a simulation must be run until it achieves quasi-steady state but not so long that signals

(a)

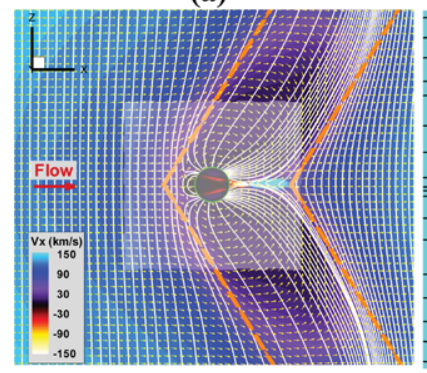

(b)

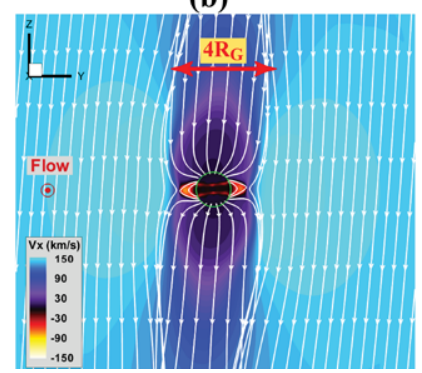

(c)

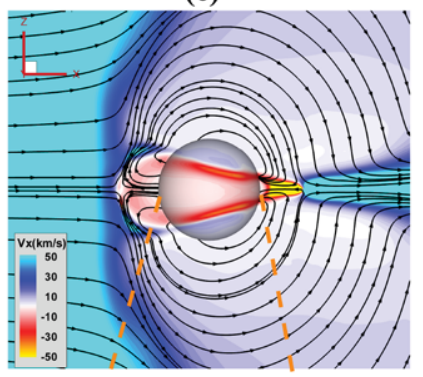

(d)

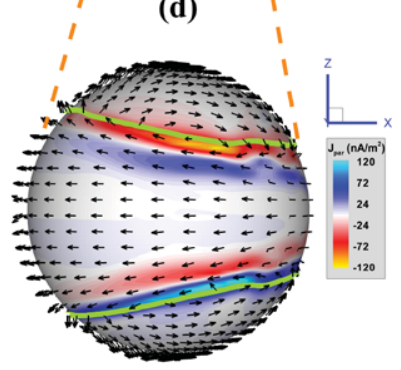

Figure 4. Figure from Jia et al. (2009a). (a) Flows and the projection of field lines (white solid lines) in the $X Z$ plane at $Y=0$. Color represents the $V_{x}$ contours and unit flow vectors in yellow show flow direction. A theoretical prediction of the Alfvén characteristics (orange dashed lines) is shown for reference. The projection of the ionospheric flow also is shown as color contours on a circular disk of $r=1.08 \mathrm{R}_{G}$ in the center. (b) As in (a) but in the $Y Z$ plane at $X=0$. (c) A zoom-in of the highlighted area in (a). Flow streamlines are superposed with the color contours of $V_{x}$. Note a different color bar is used to better illustrate the relatively weak flow within the magnetosphere. (d) Field-aligned current density along with unit flow vectors shown on a sphere of radius $\mathrm{r}=1.08 \mathrm{R}_{G}$. 
return from the boundaries of the simulation space. The boundary conditions at the moon are not obvious, but systematic analysis of a range of boundary conditions can convincingly identify plausible results and improve the match to data.

The general form of the magnetic field data measured on several passes through Ganymede's magnetosphere can be obtained from the multifluid simulation of Paty and Winglee (2006). Their assertion that a multifluid treatment is needed to obtain good fits is contradicted by the results of the MHD simulation of Jia (2009). That simulation not only more closely represents the magnetic field measured on all passes by Ganymede, but also gives a good account of the plasma flow measurements. Focusing on the Jia simulation, we next comment on the assumptions and the modifications introduced that led to the desired good agreement with measurements.

The 3D MHD simulation model of Jia et al. The Jia (2009) simulation

- uses a resistive MHD code;

- uses a spherical grid that provides very high resolution near the moon, but generates unwanted signals where the spherical grid encounters the Cartesian downstream boundary;

- is run until it is quasi-stable but cut off before the signals get back to the interaction region;

- tests various inner boundary conditions (at $1.05 \mathrm{R}_{G}$ ) that differ in rather subtle ways but produce very different results. The acceptable inner boundary conditions are required to produce physically plausible flows and to predict field and plasma configurations consistent with observations.

The code employs a $131 \times 132 \times 128 \times(r, \theta, \phi)$ non-uniform spherical mesh covering a calculation domain $0.5 R_{G}<r<40 R_{G}$ with the grids $\left(\sim 0.02 R_{G}\right.$ or $50 \mathrm{~km}$ in characteristic lenght) near Ganymede. The resolution was increased in regions near the low

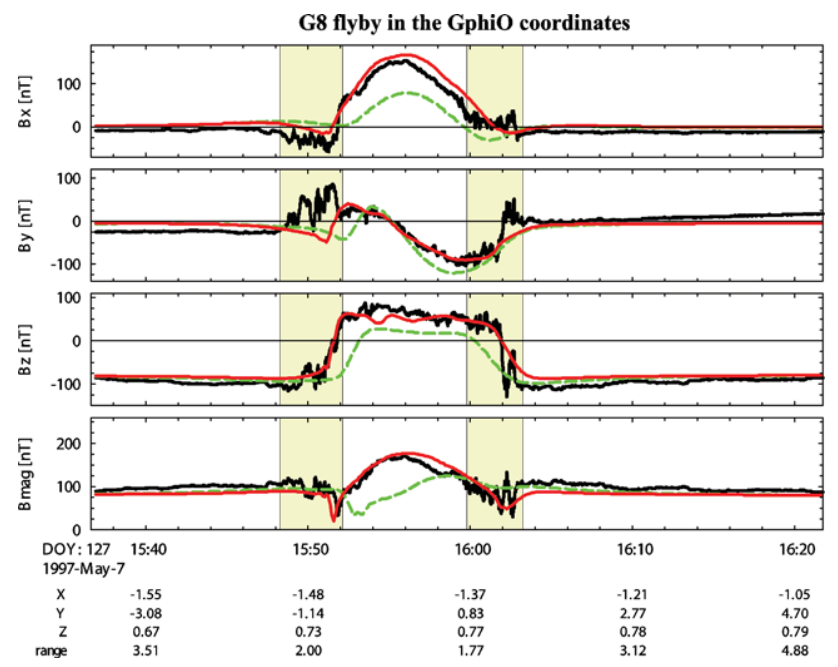

Figure 5. Figure from Jia et al. (2009a). Magnetic field comparisons between the simulation results and the Galileo observations for the G8 flyby. Black solid lines are the spacecraft measurements, green dashed lines are results extracted from the fixed boundary condition run and red solid lines are from the boundary condition allowing finite flow transverse to $\mathbf{B}$. The locations where large amplitude magnetic fluctuations are observed during both inbound and outbound magnetopause crossings are marked with shading and have been found to correspond to regions with large temporal fluctuations in the simulation. 
latitude upstream magnetopause boundary until the simulation reproduced the rapid field reversal observed as Galileo crossed that region. With inadequate spatial resolution, neither the duration nor the field magnitude on the two sides was properly reproduced.

The non-linear, coupled equations solved in the analysis are given in Jia et al. (2008). They include continuity, momentum, and energy density and allow for sources and sinks of ions. The magnetic field is derived from a vector potential that satisfies Faraday's law and Ampere's law is used to derive the current density from the curl of the field. Although the code in principle allows for a neutral source, the effects of such a source are not of great importance at Ganymede and have not been included.

Inner boundary conditions In matching the simulation results to the observations, Jia found that the solutions are extremely sensitive to the boundary conditions imposed on the plasma velocity at the inner boundary (Ganymede's ionosphere). In testing alternative ways of handling those boundary conditions, he focused on pass G8, an upstream pass at mid-Ganymede latitude. This pass was selected because the trajectory passed relatively close to Ganymede in a region also close to strong currents of the magnetopause, a particularly hard part of the system to model quantitatively. Inner boundary conditions (at $1.05 \mathrm{R}_{G}$ ) were tweaked until the magnetic field signature for pass G8 gave good agreement with the magnetometer data. Various familiar and seemingly plausible assumptions were tested: no flow (appropriate for a highly conducting obstacle), vanishing normal flow (plasma cannot penetrate the boundary) and either continuity of tangential flow or continuity of flow perpendicular to $\mathbf{B}$ (the latter form being consistent with equipotential field lines). Only the latter boundary conditions gave output that was consistent with the spacecraft data, and in particular gave a good correspondence to the observed location of the magnetopause.
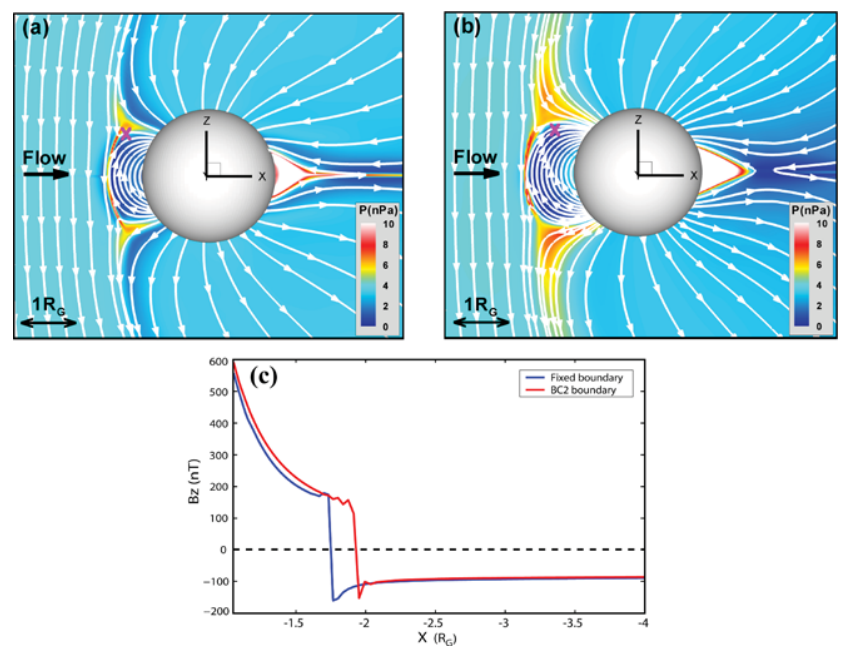

Figure 6. Figure from Jia et al. (2009a). Comparison of the magnetospheric configuration for (a) no flow at the ionospheric boundary and (b) no flow along $\mathbf{B}$ and continuous flow perpendicular to $\mathbf{B}$ at the inner boundary. In each plot, projected field lines are shown in white in the $X Z$ plane at $Y=0$ superposed with color contours of plasma thermal pressure. The intersection of the spacecraft trajectory with the $X Z$ plane is represented by the magenta cross near the northern cusp. The inner boundary of the simulation is represented as a centered sphere. (c) The $B_{z}$ component traced along the $-X$-axis on the upstream side (not along Galileo's trajectory): blue line for boundary conditions as in (a) and red line for boundary conditions as in (b). 
Simulation results Adopting the inner boundary conditions consistent with equipotential field lines and making some additional adjustments to permit nonlinear increases of the resistivity in regions of large field gradients, Jia obtained extremely satisfactory agreement with the data for Galileo's G8 pass on May 7, 1997 as illustrated in Figure 5 . The new treatment of resistance facilitates reconnection in the vicinity of the magnetopause and in the downstream return flow region. The large amplitude fluctuations in the data taken in the vicinity of the magnetopause crossings correspond to signatures of intermittent reconnection in the simulation, as evident from movies of the time series simulation data, so they are not likely to be either boundary waves (as suggested by Kivelson et al., 1998) or spatial structures.

Changes of flow patterns associated with different boundary conditions are accompanied by changes in the shape and size of the magnetosphere; this is illustrated in Figure 6 , a cut along the meridian containing the upstream flow. The appropriate boundary conditions enlarge the magnetosphere and move the magnetopause away from Ganymede.

Having developed a model that corresponds well with the magnetic field data from the G8 pass, Jia ran the simulations with no further changes other than to represent the upstream conditions appropriately for the remaining 5 Galileo passes by Ganymede. In all cases, the correct boundary conditions improved the fits and the simulated results gave a close approximation to the smoothed magnetometer data from all passes. (We believe that the fluctuations present in the actual data result from temporal fluctuations produced by intermittent reconnection occurring during the passes.)

Interpretations of the plasma data in regions of low flow velocity require assumptions about the mass per unit charge of the plasma ions. There has been some dispute as to whether the dominant ions within Ganymede's magnetosphere are protons (Frank et al., 1997) or oxygen (Eviatar et al., 2001). Jia's runs provide excellent models of plasma properties, as shown in Figure 7 for the G2 pass of Sept. 6, 1996, if ions are taken as oxygen.

In summary, the simulations developed by Jia (Jia et al, 2008, 2009a; Jia, 2009) provide excellent fits to data on both the magnetic field and the plasma within Ganymede's magnetosphere. They serve the objective desired for improving fits to the internal field of Ganymede.

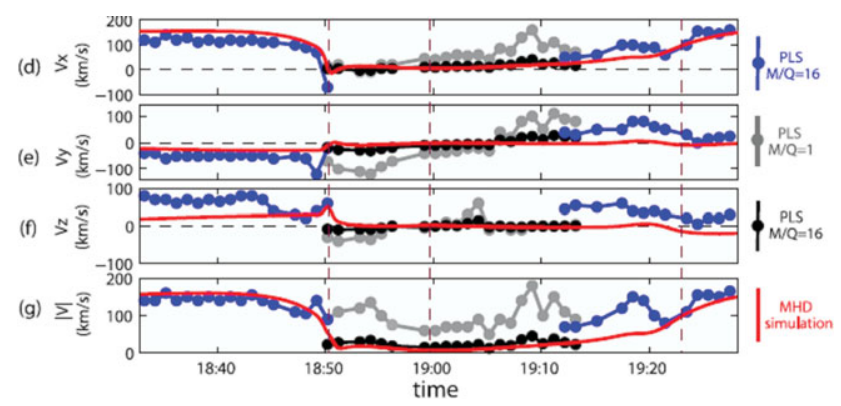

Figure 7. Figure from Jia et al. (2009a). Flow velocity inferred by the plasma investigation for the G2 pass of Sept. 6, 1996 with gray (black) dots relevant if ions are protons $\left(\mathrm{O}^{+}\right)$(Frank et al., 1997). Red curves are flow velocity from the Jia simulation. 


\section{Improved estimates of the internal field of Ganymede}

Jia's simulation uses the best previous model of the internal field of Ganymede and adds to it a flowing plasma threaded by a uniform field representing the moon's surroundings in the Jovian magnetosphere. The output of the simulation differs from the input because of perturbation fields and their associated plasma currents. Thus, the difference between the output and the total input field (background field plus the assumed internal field) at each grid cell is taken to represent the contribution to the total field from currents driven through the plasma. By removing the sum of the modeled difference field and the input uniform field from the measured field, one obtains the approximate signature of the internal field along the spacecraft trajectory. The internal field should not vary over the few years of the Galileo encounters, so the difference signatures from all passes can be combined to obtain a model of the internal field. Evidently, if the new model differs markedly from that assumed in the simulation, the process should be iterated, but for the case of Ganymede, the changes between the assumed internal field and the new fit is rather small and the process has not been iterated.

Figure 8 (Jia, 2009) gives the model internal field obtained by fitting the difference signature from 6 passes to dipole plus quadrupole moments ( 8 independent parameters) or, alternatively, by fitting a permanent dipole (the same for all passes) plus an inductive response that varies with the phase of Jupiter's rotation and therefore differs from pass to pass ( 4 independent parameters). If there is a significant conducting layer, the inductive response can be characterized in terms of a single parameter, an efficiency factor, i.e., the fraction of the maximum response that would be found if the moon were a perfect conductor of radius $r_{\text {moon }}$.

Examining the table, one sees that:

- the new models differ rather little from the previously published ones (MK02, Kivelson et al., 2002). However, the new models reduce induction efficiency compared with the earlier estimates;

- all new models give nearly same dipole moment: $\mathrm{B}_{\text {suf }}=728 \mathrm{nT}(719 \mathrm{nT})$, tilt $=$ $177^{\circ}$ or $176^{\circ}$, rotation $\sim 4^{\circ}$ or $\sim 24^{\circ}$ from the Jupiter-facing meridian;

- the ratio of quadrupole to dipole moments is small;

- the multipole model and the dipole plus induction model represent the data equally well, but the former requires fewer free parameters. Although the new fits were obtained by a refined process, they still do not enable us to establish the presence of an inductive response with any more certainty than did earlier treatments.

If the induced field is present, the relatively large value of induction efficiency listed in the table requires both that the conducting layer lie not far below the surface, well

\begin{tabular}{|c|c|c|c|c|c|c|c|c|c|c|c|c|c|c|c|}
\hline & \multirow[b]{2}{*}{ Model } & \multicolumn{3}{|c|}{ Dipole coefficients } & \multirow[b]{2}{*}{ Ind } & \multicolumn{5}{|c|}{ Quadrupole coefficients } & \multicolumn{5}{|c|}{ rms error } \\
\hline & & $g_{1}^{0}$ & $g_{1}^{1}$ & $h_{1}^{1}$ & & $g_{2}^{0}$ & $g_{2}^{1}$ & $g_{2}^{2}$ & $h_{2}^{1}$ & $h_{2}^{2}$ & G1 & $\mathrm{G} 2$ & G8 & G28 & total \\
\hline Dipole & MKO2 & -716.8 & 49.3 & 22.2 & 0.84 & & & & & & 9.8 & 7.5 & & 15.6 & 11.5 \\
\hline+ & SimModel1 & \begin{tabular}{|l|}
-726.3 \\
\end{tabular} & 39.2 & 5.0 & 0.63 & & & & & & 11.9 & 10.4 & & 10.6 & 11.1 \\
\hline Induction & SimModel3 & -725.8 & 39.7 & 6.6 & 0.63 & & & & & & 11.6 & 10.8 & 11.3 & 10.7 & 11.1 \\
\hline Dipole & MKO2 & -711.0 & 46.8 & 22.3 & & 0.9 & 27 & -0.4 & 1.8 & -11 & 11.9 & 15.6 & & 13.2 & 13.5 \\
\hline+ & SimModel2 & -729.2 & 42.9 & -0.6 & & 4.3 & 6.1 & -5.7 & 8.0 & -23.7 & 10.4 & 10.0 & & 9.8 & 10.1 \\
\hline Quadrupole & SimModel4 & -727.9 & 43.4 & 1.0 & & 3.3 & 7.0 & -6.6 & 8.1 & -21.8 & 10.4 & 10.5 & 11.9 & 9.5 & 10.4 \\
\hline
\end{tabular}

Figure 8. Parameters of internal field models for Ganymede from Jia (2009). Ind denotes the induction efficiency 
within the $800 \mathrm{~km}$ of ice that forms the outer shell, and that the conductivity be of order $1 \mathrm{~S}$. The conductivity of ice is not sufficiently high to produce a response as large as $63 \%$ of the maximum possible response. However, a liquid layer buried within the ice layer could well be expected to have conductivity close to or somewhat below that of terrestrial sea water $(2.5 \mathrm{~S})$. The data do not constrain the precise depth of the ocean below the surface nor the precise conductivity, but reasonable combinations can be made consistent with the known thickness of the ice shell, probable thermal profiles, and sensible values of ocean conductivity.

\section{Summary and a glimpse into future developments}

Although the interaction of the Jovian plasma with the Medicean moons is itself of considerable scientific interest, this chapter describes the interactions mainly to emphasize that they compromise the reliable identification of the internal field of a moon, whether permanent or induced, from the field measured along spacecraft trajectories. An approach, recently developed, characterizes the perturbation of the field by use of a well calibrated MHD simulation. The perturbation field inferred from the simulation plus the unperturbed external field are subtracted from the measured field, yielding a good approximation to the field that would be measured along the spacecraft trajectory in the absence of external sources.

Using data from all the passes by Ganymede, the differenced field has been modeled either as a permanent internal field with dipole and quadrupole components or a permanent dipole plus an inductive response to the driving field imposed from Jupiter's magnetosphere. The inductive response is characterized by the efficiency of the inductive response, a parameter that depends on the conductivity of the responding layer and its depth. The inductive response model uses only 4 free parameters, but achieves a fit that is as good as the fit to the 8 parameters of the dipole plus quadrupole model. Although one cannot rule out the possibility that there is no inductive response, the rule of economy is appealing and it seems plausible that the inductive response is real. The new fits reduce the response efficiency from 0.83 previously suggested to 0.63 (see Figure 8 ). This level of response can be understood if the conducting layer is a buried ocean (at a depth of about $150 \mathrm{~km}$ ) with conductivity lower than seawater. Elsewhere (Jia et al., 2010) the implications of the imperfect response is discussed in greater detail.

The fact that the internal fields of Ganymede, Europa, and Callisto can be fit quite well by assuming inductive responses to the time-variations of the field imposed upon them is consistent with there being global shells of conductivity somewhere within their icy outer layers. For these icy moons, the conducting material is likely to be a buried liquid water ocean. Io is known to hide molten lava beneath its surface. Conducting magma is a plausible source of an inductive signature. Models of the internal field of Io based on the field measured in Galileo flybys of Io have not previously considered the possibility of an inductive response. However, Khurana, Jia, and Kivelson (2010) are using the technique described here for the case of Ganymede to examine Io's response to the time-varying field of Jupiter. The conductivity of melted magma is high enough to sustain an inductive response if the layer is thick enough and forms a nearly complete spherical shell. It may turn out that molten shells are ubiquitous among the Medicean moons, but confirmation must await additional analysis. 


\section{References}

Anderson, J. D., Lau, E. L., Shogren, W. L., Schubert, G., \& Moore, W. B. 1996, Nature, 384 Busse, F. H. 1976, Phys. of the Earth and Plan. Int., 12, 350

Eviatar, A., Vasyliunas, V. M., \& Gurnett, D. A. 2001, Planet. Space Sci., 49, 327

Frank, L. A., Paterson, W. R., Ackerson, K. L., \& Bolton, S. J. 1997, Geophys. Res. Lett., 24, 2,151

Jia, X., Walker, R. J., et al. 2008, J. Geophys. Res., 113, A06212, doi:10.1029/2007JA012748

Jia, X. 2009, Ph.D. Dissertation, Dept. of Earth and Space Sciences, UCLA

Jia, X., Walker, R. J., Kivelson, M. G., Khurana, K. K., \& Linker, J. A. 2009a, J. Geophys. Res., 114, doi:10.1029/2009JA014375

Jia, X., Kivelson, M. G., Khurana, K. K., \& Walker, R. J. 2009b, Space Sci Rev, doi:10.10.1007/s11214-009-9507-8

Jia, X., Walker, R. J., Kivelson, M. G., Khurana, K. K., \& Linker, J. A. 2010, J. Geophys. Res., doi:10.1029/2010JA015771

Khurana, K. K., Kivelson, M. G., et al. 1998, Nature, 395, 777

Khurana, K. K., Jia, X., \& Kivelson, M. G. 2010, A magma ocean at Io?, in preparation

Kivelson, M. G. 2004, Adv. Space Res., 33, 2061

Kivelson, M. G., Khurana, K. K., et al. 1996, Nature, 384, 537

Kivelson, M. G., Khurana, K. K., Russell, C. T., \& Walker, R. J. 1997, Geophys. Res. Lett., 24, 2127

Kivelson, M. G., Khurana, K. K., et al. 1997, Geophys. Res. Lett., 24, 2155

Kivelson, M. G. et al. 1998, J. Geophys. Res., 103, 19963

Kivelson, M. G., Khurana, K. K., et al. 2001, J. Geophys. Res., 106, 26, 121

Kivelson, M. G., Khurana, K. K., \& Volwerk, M. 2002, Icarus, 157, 507

Kivelson, M. G., Bagenal, F., et al. 2004, Chapter 21 in Jupiter: The Planet, Satellites and Magnetosphere edited by F. Bagenal, T. Dowling and W. McKinnon, Cambridge University Press

Neubauer, F. M. 1978, Geophys. Res. Lett., 5, 905

Paty, C. \& Winglee, R. 2006, Geophys. Res. Lett., 33, L10106, doi:10.1029/2005GL025273

Saur, J., Strobel, D. F., \& Neubauer, F. M. 1998, J. Geophys. Res., 103, 19, 947

Schilling, N., Neubauer, F. M., \& Saur, J. 2007, Icarus, 192, 41

Schilling, N., Neubauer, F. M., \& Saur, J. 2008, J. Geophys. Res., 113, A03203.doi:10.1029/2007JA012842

Schubert, G., Stevenson, D. J., \& Ellsworth, K. 1981, Icarus, 47, 46

Showmann, A. P. \& Malhotra, R. 1997, Icarus, 127, 93

Walker, R. J. \& Russell, C. T. 1995, in Introduction to Space Physics edited by Kivelson, M. G., \& Russell, C. T., New York, Cambridge U. Press

Zimmer, C., Khurana, K. K., \& Kivelson, M. G. 2000, Icarus, 147, 329 